\title{
Upper versus lower body forced-air warming to prevent hypothermia during thoracoscopic surgery in the lateral decubitus position : a randomised controlled trial
}

Sehee Yoon, Se-Hee Min, Susie Yoon, Jae-Hyon Bahk, Jeong-Hwa Seo

Department of Anaesthesiology and Pain Medicine, Seoul National University Hospital

\section{Background}

- To prevent hypothermia during abdominal or thoracic surgery, forced-air warming can be applied to the upper or lower bodies not involved in the surgical area.

- In the supine position, lower body warming increases the core temperature more effectively than upper body warming since it covers a larger body surface area.

- However, it is unknown in the lateral decubitus position.

\section{Methods}

\section{* Subjects}

Patients were randomised to receive forced-air warming on the upper body or lower body during thoracoscopic surgery in the lateral position.

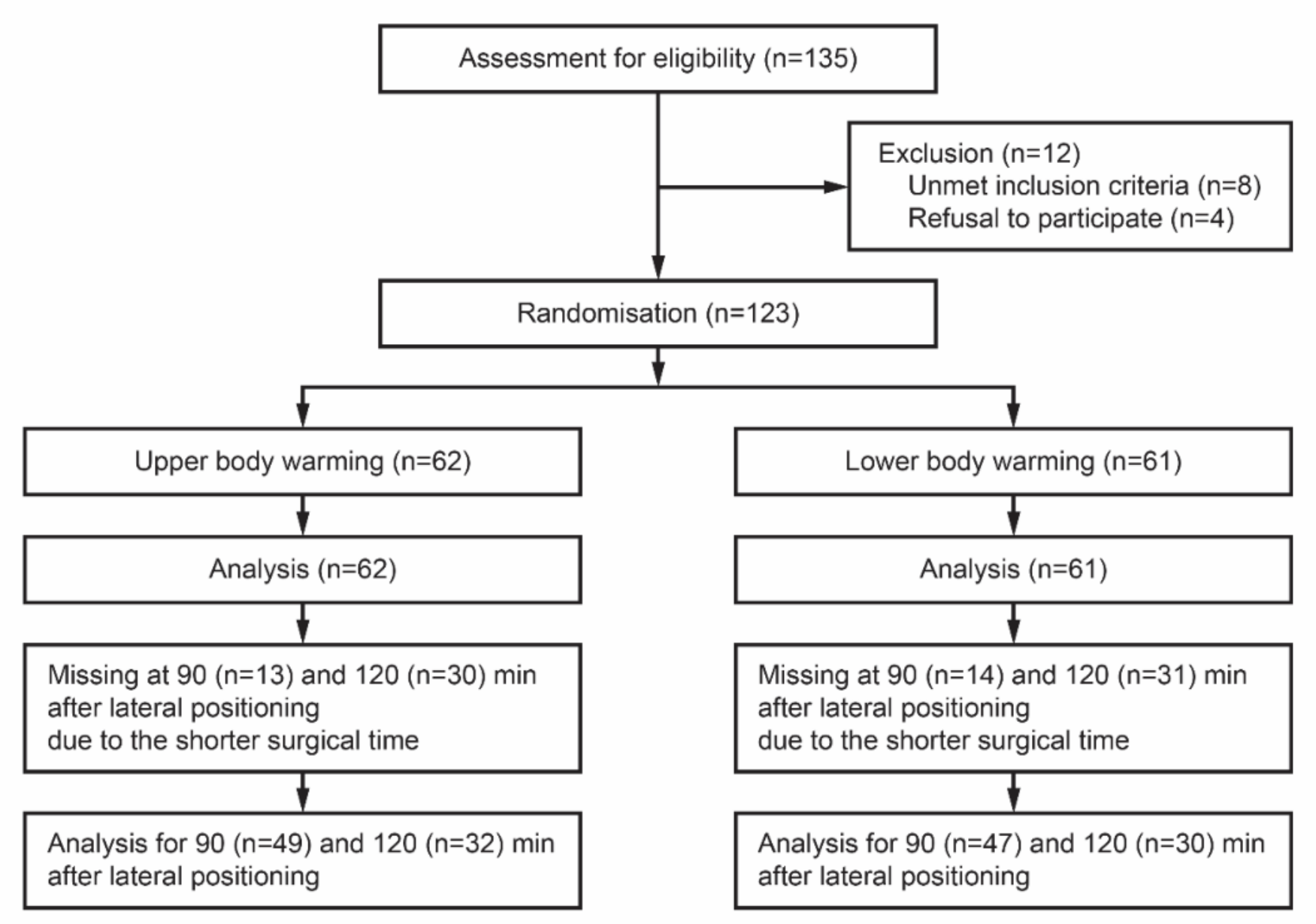

\section{* Study Protocol}

- The ambient temperature of the OR was $22 \pm 1^{\circ} \mathrm{C}$, and a mattress was heated with circulating water set to $40^{\circ} \mathrm{C}$.

- A disposable thermistor was positioned in the posterior upper nasopharynx.

- After lateral positioning, a disposable upper body blanket and lower body blanket was applied, and two forced-air blowers were connected to each blanket.

- One of two, according to assignment group, was set to temperature of $43^{\circ} \mathrm{C}$ for warming.

- Patients received both upper and lower body warmings when a temperature is below $35.5^{\circ} \mathrm{C}$.
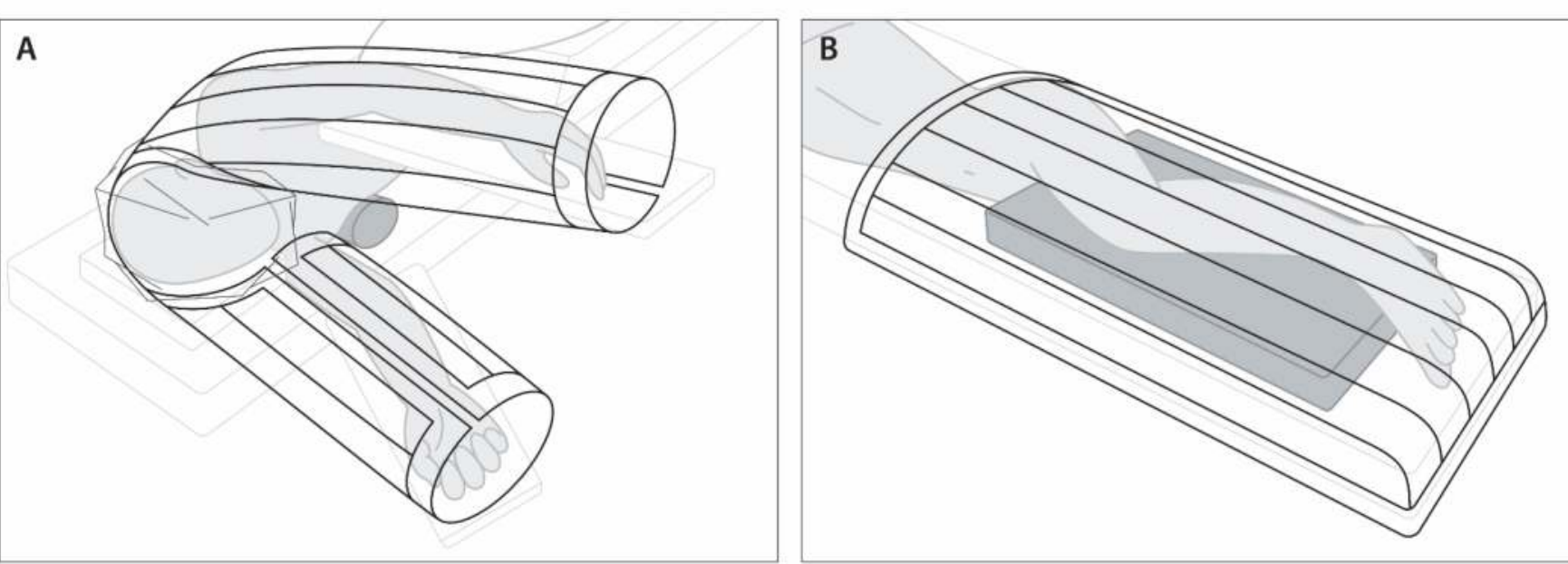

\section{* Outcomes}

- The primary outcome was the incidence of intraoperative hypothermia with a temperature of $<36.0^{\circ} \mathrm{C}$.

- During surgery, the nasopharyngeal temperatures were

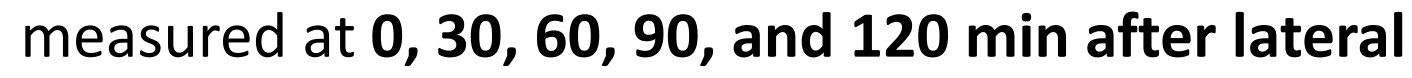
positioning, and the infrared tympanic membrane temperatures were measured at $\mathbf{0 , 3 0 , 6 0 , 9 0}$ and $120 \mathrm{~min}$ after surgery.

- Postoperative shivering, thermal discomfort, and pain intensity were assessed.

\section{Results}

- The overall incidence of intraoperative hypothermia was lower with the upper body warming than with the lower body warming [21/62 vs 35/61, risk ratio $(95 \% \mathrm{Cl}) 0.6(0.4-0.9)$, $\mathrm{P}=0.011]$.

- Fewer patients received both upper and lower body warmings due to a temperature of $\angle 35.5^{\circ} \mathrm{C}$ in the upper body warming group [1/62 vs 7/61, $\mathrm{P}=0.032$ ]

- The intraoperative temperature was higher with the upper body warming than with the lower-body warming at 30, 60, and 90 min after lateral positioning.
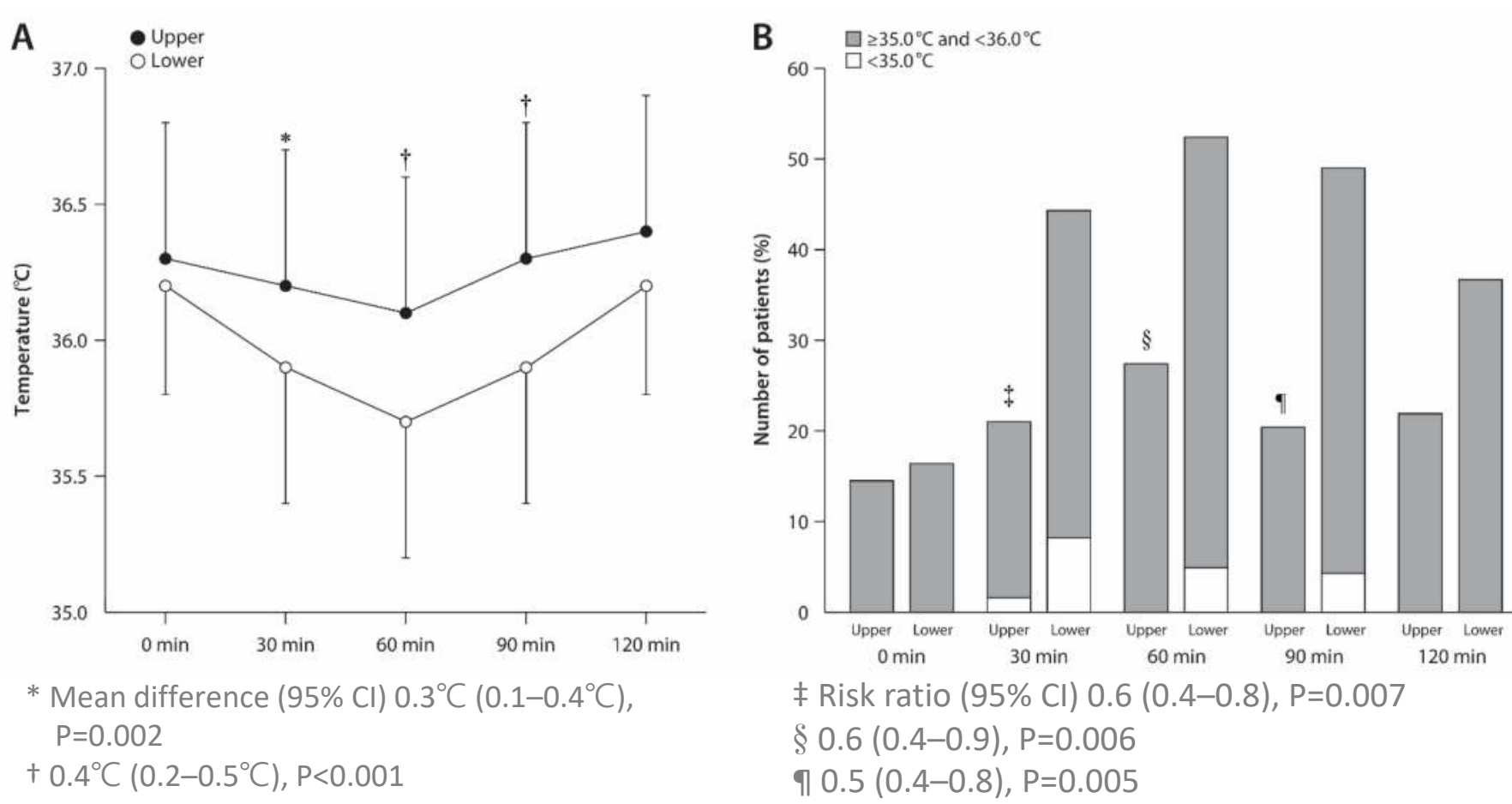

- The postoperative infrared tympanic membrane temperature was higher in the upper body warming group than in the lower body warming group at 0 min and 30 min after surgery.

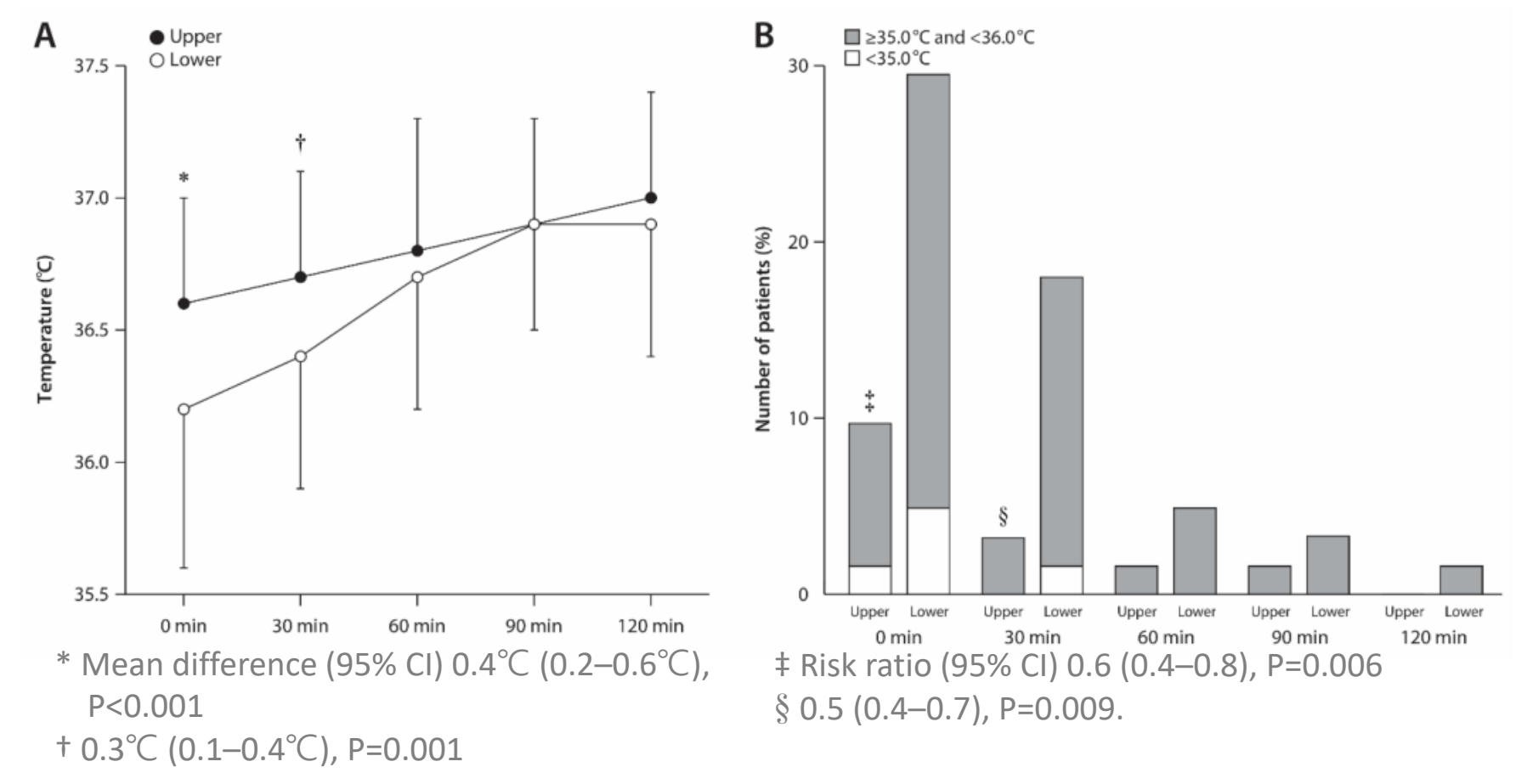

- No differences were found in postoperative shivering, thermal discomfort, recovery time, adverse events, and pain intensity.

\section{Conclusions}

- Forced-air warming was more effective on the upper body than on the lower body to prevent hypothermia during thoracoscopic surgery in the lateral decubitus position. 